\title{
Analysis of Probability of Non-zero Secrecy Capacity for Multi-hop Networks in Presence of Hardware Impairments over Nakagami- $m$ Fading Channels
}

\author{
Tran Tin PHU ${ }^{1,2}$, The Hung DANG ${ }^{3}$, Trung Duy TRAN ${ }^{4}$, Miroslav VOZNAK ${ }^{1}$ \\ ${ }^{1}$ VSB Technical University of Ostrava, 17. listopadu 15/2172, 70833 Ostrava - Poruba, Czech Republic \\ ${ }^{2}$ Faculty of Electronics Technology, Industrial University of Ho Chi Minh City, Ho Chi Minh City, Vietnam \\ ${ }^{3}$ Faculty of Telecommunications, Telecommunications University, Nha Trang City, Vietnam \\ ${ }^{4}$ Department of Telecommunications, Posts and Telecommunications Institute of Technology, Ho Chi Minh City, Vietnam
}

phutrantin79@gmail.com,danghung8384@gmail.com, trantrungduy@ptithcm.edu.vn,miroslav.voznak@vsb.cz

Manuscript received May 19, 2016

\begin{abstract}
In this paper, we evaluate probability of non-zero secrecy capacity of multi-hop relay networks over Nakagami$m$ fading channels in presence of hardware impairments. In the considered protocol, a source attempts to transmit its data to a destination by using multi-hop randomize-and-forward $(R F)$ strategy. The data transmitted by the source and relays are overheard by an eavesdropper. For performance evaluation, we derive exact expressions of probability of non-zero secrecy capacity (PoNSC), which are expressed by sums of infinite series of exponential functions and exponential integral functions. We then perform Monte Carlo simulations to verify the theoretical analysis.
\end{abstract}

\section{Keywords}

Hardware impairments, probability of non-zero secrecy capacity, multi-hop networks, Nakagami- $m$ fading channel

\section{Introduction}

Recently, physical-layer security (PLS) has gained much attention as an efficient method to obtain the security in the presence of eavesdroppers, without using complex cryptographic methods [1]. In PLS, the performance is measured by secrecy capacity which is different between channel capacity of the data and eavesdropping links [2]. In addition, important performance metrics such as secrecy outage probability (SOP) and probability of non-zero secrecy capacity (PoNSC) are commonly used to evaluate the system secrecy performance [3].

To improve the secrecy performances for wireless systems, diversity relaying protocols [4], [5] have become a promising technique. In [6] and [7], the authors evaluated the secrecy performances of the relaying networks at the second phase (cooperative phase). In particular, the pub- lished work [6] proposed the joint relay and jammer selection methods to enhance the security for secondary networks in underlay cognitive radio. The authors in [7] investigated the impact of co-channel interference on the secrecy performance of various relay selection strategies. In [8] and [9], dual-hop secured communication protocols in one-way and two-way relaying networks were studied, respectively. Moreover, the authors in [8] proposed a switch-and-stay combining method to obtain the security, while the authors in [9] investigated impact of antenna selection on two-way secured communication scenarios. Published works [10], [11] focused on the secured transmission in multi-hop approaches. In [10], cluster-based multi-hop protocols with various relay selection methods applied for each dual-hop were proposed to further improve the secrecy performances, as compared with the random and conventional relay selection methods. The authors in [11] considered the impact of the positions and transmit power of the interference sources (external sources) on the SOP in the limited interference environment.

However, the authors in [10], [11] assumed that transceiver hardware of wireless devices is perfect. In practice, the transceiver hardware is imperfect due to phase noises, amplifier-amplitude non-linearity and in phase and quadrature imbalance (IQI) [12], [13], which degrades performances of wireless relay networks. The authors in [14] studied the effects of the IQI on the secrecy capacity of the wiretap channel. Results in [14] presented that the IQI should be taken into account in the design of secured communication systems.

To the best of our knowledge, only the published work [14] evaluating the secrecy performances in the presence of the hardware imperfection. However, the authors in [14] only considered the effects of the IQI in one-hop orthogonal frequency-division multiple access (OFDMA) communication systems. Unlike [14], this paper investigates the impact of hardware impairments on the probability of non-zero se- 
crecy capacity (PoNSC) of a multi-hop transmission scheme, where the data transmitted from a source, via multiple relays, to a destination is overheard by an eavesdropper. In the proposed scheme, the transceiver hardware of the source, relays, destination and eavesdropper is not perfect. Furthermore, the randomize-and-forward (RF) strategy is used by the source and relays so that the eavesdropper cannot combine the received data [15]. For performance evaluation, we derive exact expressions of the PoNSC over Nakagami- $m$ fading channels, which are expressed by sums of infinite series of exponential functions and exponential integral functions. In order to provide insights into the system performance, asymptotic closed-form expressions of the PoNSC at high transmit signal-to-noise ratio (SNR) are also provided. Finally, Monte Carlo simulations are performed to verify the theoretical derivations.

The rest of this paper is organized as follows. The system model of the proposed protocol is described in Sec. 2. In Sec. 3, we evaluate the performance of the considered protocol. The simulation results are shown in Sec. 4. Finally, this paper is concluded in Sec. 5 .

\section{System Model}

In Fig. 1, we present the system model of the proposed protocol, where the source $\mathrm{T}_{0}$ attempts to transmit its data to the destination $\mathrm{T}_{N}$ via $N-1$ relay nodes, i.e., $\mathrm{T}_{1}, \mathrm{~T}_{2}, \ldots, \mathrm{T}_{N-1}$. In this network, there is an eavesdropper $\mathrm{E}$ overhearing the data transmitted by the source and relays. We assume that all of the nodes are equipped with a single antenna; therefore, the data transmission is realized by time division multiple access (TDMA). Moreover, the transmitting nodes such as source and relays employ the randomize-and-forward (RF) technique to confuse the eavesdropper. In particular, the source and relays encode the data by randomly generating code-books to avoid the eavesdropper $\mathrm{E}$ from combining the received data [15]. We also assume that the eavesdropper $\mathrm{E}$ is passive and hence the channel state information (CSI) of the eavesdropping links are not available at the authorized nodes. However, the statistics such as path-loss exponent, link distances between nodes, distributions of the wireless channels are assumed to be available.

Let us denote $h_{n}$ and $g_{n}$ as channel gains of the $\mathrm{T}_{n-1} \rightarrow \mathrm{T}_{n}$ and $\mathrm{T}_{n-1} \rightarrow \mathrm{E}$ links, respectively, where $n=1,2, \ldots, N$. It is assumed that all of the fading channels between two arbitrary terminals are Nakagami- $m$ [16]. The Nakagami- $m$ fading channel is a generalized model which is widely used to describe different fading environments.

Considering the communication at the $n$th hop between the nodes $\mathrm{T}_{n-1}$ and $\mathrm{T}_{n}$, the instantaneous $\mathrm{SNR}$ received at $\mathrm{T}_{n}$ can be given as in [12, eq. (17)]:

$$
\begin{aligned}
\Psi_{\mathrm{D} n} & =\frac{P h_{n}}{\left(\kappa_{t, \mathrm{D} n}^{2}+\kappa_{r, \mathrm{D} n}^{2}\right) P h_{n}+N_{0}}=\frac{\psi h_{n}}{\kappa_{\sum, \mathrm{D} n}^{2} \psi h_{n}+1} \\
& =\frac{\chi_{n}}{\kappa_{\mathrm{D} n} \chi_{n}+1} .
\end{aligned}
$$

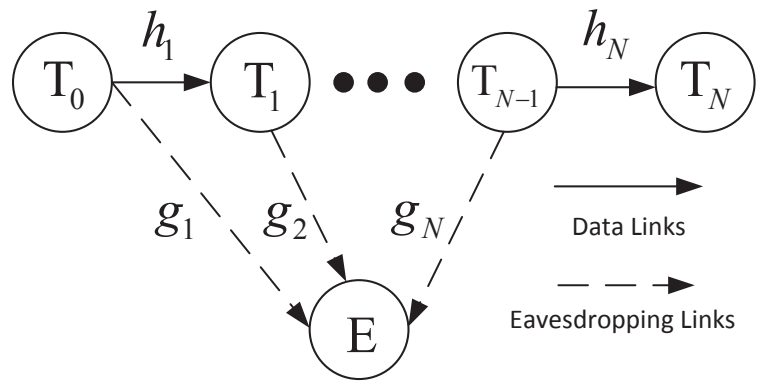

Fig. 1. System model of the proposed protocol.

In (1), $P$ is the transmit power of the node $\mathrm{T}_{n-1}$. It is assumed that all of the transmitters including the source and relays have the same transmit power. The terms $N_{0}$ in (1) is variance of zero-mean Gaussian noises at the node $T_{n}$. Next, the constants $\kappa_{t, \mathrm{D} n}$ and $\kappa_{r \text {,D } n}$ are the hardware impairment levels at the transmitter $\mathrm{T}_{n-1}$ and the receiver $\mathrm{T}_{n}$, respectively; and $\sqrt{\kappa_{\mathrm{D} n}}=\kappa_{\sum, \mathrm{D} n}=\sqrt{\kappa_{t, \mathrm{D} n}^{2}+\kappa_{r, \mathrm{D} n}^{2}}$ is the aggregate level of impairments on the $\mathrm{T}_{n-1} \rightarrow \mathrm{T}_{n}$ link [12]. Finally, the remaining notations in (1) are denoted as follows: $\psi=P / N_{0}$ and $\chi_{n}=\psi h_{n}$.

Similarly, the SNR received at the eavesdropper E from the transmission of the node $\mathrm{T}_{n-1}$ is expressed by

$$
\Psi_{\mathrm{E} n}=\frac{P g_{n}}{\left(\kappa_{t, \mathrm{D} n}^{2}+\kappa_{r, \mathrm{E}}^{2}\right) P g_{n}+N_{0}}=\frac{\varphi_{n}}{\kappa_{\mathrm{E} n} \varphi_{n}+1}
$$

where $\kappa_{r, \mathrm{E}}$ is the level of the hardware impairments at the eavesdropper $\mathrm{E} ; \kappa_{\mathrm{E} n}=\kappa_{t, \mathrm{D} n}^{2}+\kappa_{r, \mathrm{E}}^{2} ;$ and $\varphi_{n}=\psi g_{n}$.

Because $h_{n}$ and $g_{n}$ are channel gains of Nakagami- $m$ fading channels, their probability density functions (PDFs) can be given respectively as (see [17, eq. (7)])

$$
\begin{aligned}
& f_{h_{n}}(x)=\frac{1}{\Gamma\left(m_{\mathrm{D} n}\right)}\left(m_{\mathrm{D} n} \sigma_{n}\right)^{m_{\mathrm{D} n}} x^{m_{\mathrm{D} n}-1} \exp \left(-m_{\mathrm{D} n} \sigma_{n} x\right), \\
& f_{g_{n}}(x)=\frac{1}{\Gamma\left(m_{\mathrm{E} n}\right)}\left(m_{\mathrm{E} n} \tau_{n}\right)^{m_{\mathrm{E} n}} x^{m_{\mathrm{E} n}-1} \exp \left(-m_{\mathrm{E} n} \tau_{n} x\right)
\end{aligned}
$$

where $m_{\mathrm{D} n}$ and $m_{\mathrm{E} n}$ are Nakagami- $m$ parameters of the $\mathrm{T}_{n-1} \rightarrow \mathrm{T}_{n}$ and $\mathrm{T}_{n-1} \rightarrow$ E links, respectively; $\sigma_{n}=1 / \mathcal{E}\left\{h_{n}\right\}$ and $\tau_{n}=1 / \mathcal{E}\left\{g_{n}\right\}(\mathcal{E}(X)$ is expected value of $\mathrm{RV} X$ $\left.\left(X \in\left\{h_{n}, g_{n}\right\}\right)\right)$; and $\Gamma($.$) is Gamma function [18].$

Moreover, since $\chi_{n}=\psi h_{n}$ and $\varphi_{n}=\psi g_{n}, \chi_{n}$ and $\varphi_{n}$ are also Nakagami- $m$ random variables (RVs). When $m_{\mathrm{D} n}$ and $m_{\mathrm{E} n}$ are integers, the PDFs of the RVs $\chi_{n}$ and $\varphi_{n}$ can be obtained as (see [19, eq. (4)])

$$
\begin{aligned}
f_{\chi_{n}}(x) & =\left(\frac{m_{\mathrm{D} n} \sigma_{n}}{\psi}\right)^{m_{\mathrm{D} n}} \frac{x^{m_{\mathrm{D} n}-1}}{\left(m_{\mathrm{D} n}-1\right) !} \exp \left(-\frac{m_{\mathrm{D} n} \sigma_{n}}{\psi} x\right) \\
& =\frac{\lambda_{n}^{m_{\mathrm{D} n}} x^{m_{\mathrm{D} n}-1}}{\left(m_{\mathrm{Dn}}-1\right) !} \exp \left(-\lambda_{n} x\right), \\
f_{\varphi_{n}}(x) & =\left(\frac{m_{\mathrm{E} n} \tau_{n}}{\psi}\right)^{m_{\mathrm{E} n}} \frac{x^{m_{\mathrm{E} n}-1}}{\left(m_{\mathrm{E} n}-1\right) !} \exp \left(-\frac{m_{\mathrm{E} n} \tau_{n}}{\psi} x\right) \\
& =\frac{\Omega_{n}^{m_{\mathrm{E} n}} x^{m_{\mathrm{E} n}-1}}{\left(m_{\mathrm{E} n}-1\right) !} \exp \left(-\Omega_{n} x\right)
\end{aligned}
$$

where $\lambda_{n}=m_{\mathrm{D} n} \sigma_{n} / \psi$ and $\Omega_{n}=m_{\mathrm{E} n} \tau_{n} / \psi$. 
To take path-loss into account, we can model $\sigma_{n}$ and $\tau_{n}$, similarly to [20], as

$$
\sigma_{n}=d_{\mathrm{D} n}^{\beta}, \tau_{n}=d_{\mathrm{E} n}^{\beta}
$$

where $d_{\mathrm{D} n}$ and $d_{\mathrm{E} n}$ are distances of the $\mathrm{T}_{n-1} \rightarrow \mathrm{T}_{n}$ and $\mathrm{T}_{n-1} \rightarrow \mathrm{E}$ links, respectively; and $\beta$ is path-loss exponent which varies from 2 to 6 .

From (4), the corresponding cumulative distribution function (CDF) of the RVs $\chi_{n}$ and $\varphi_{n}$ are respectively given by

$$
\begin{aligned}
& F_{\chi_{n}}(x)=1-\exp \left(-\lambda_{n} x\right) \sum_{t=0}^{m_{\mathrm{D} n}-1} \frac{\left(\lambda_{n} x\right)^{t}}{t !}, \\
& F_{\varphi_{n}}(x)=1-\exp \left(-\Omega_{n} x\right) \sum_{t=0}^{m_{\mathrm{E} n}-1} \frac{\left(\Omega_{n} x\right)^{t}}{t !} .
\end{aligned}
$$

Now, let us consider the secrecy capacity at the $n$th hop, which can be formulated similarly as in [10]:

$$
\begin{aligned}
C_{n}^{\mathrm{Sec}} & =\max \left(0, \frac{1}{N} \log _{2}\left(1+\Psi_{\mathrm{D} n}\right)-\frac{1}{N} \log _{2}\left(1+\Psi_{\mathrm{E} n}\right)\right) \\
& =\max \left(0, \frac{1}{N} \log _{2}\left(\frac{1+\Psi_{\mathrm{D} n}}{1+\Psi_{\mathrm{E} n}}\right)\right)
\end{aligned}
$$

where the factor $1 / N$ indicates that the data transmission is split into $N$ orthogonal time slots.

Because the RF strategy is used by the source and relays, the end-to-end secrecy capacity of the $N$-hop relaying network can be obtained as

$$
C_{\mathrm{e} 2 \mathrm{e}}^{\mathrm{Sec}}=\min _{n=1,2, \ldots, N}\left(C_{n}^{\mathrm{Sec}}\right) .
$$

Equation (8) implies that the end-to-end secrecy capacity is dominated by the secrecy capacity of the weakest hop [15].

\section{Performance Analysis}

In this paper, the secrecy performance of the proposed protocol is evaluated, in terms of the PoNSC [15]. From (1), (2), (7) and (8), the PoNSC can be formulated as

$$
\begin{aligned}
\mathrm{P}_{\mathrm{NSC}} & =\operatorname{Pr}\left(C_{\mathrm{e} 2 \mathrm{e}}^{\mathrm{Sec}}>0\right)=\prod_{n=1}^{N} \operatorname{Pr}\left(C_{n}^{\mathrm{Sec}}>0\right) \\
& =\prod_{n=1}^{N} \operatorname{Pr}\left(\Psi_{\mathrm{D} n}>\Psi_{\mathrm{En}}\right) \\
& =\prod_{n=1}^{N} \underbrace{\operatorname{Pr}\left(\frac{\chi_{n}}{\kappa_{\mathrm{D} n} \chi_{n}+1}>\frac{\varphi_{n}}{\kappa_{\mathrm{E} n} \varphi_{n}+1}\right)}_{\mathrm{P}_{n}} .
\end{aligned}
$$

Moreover, the probability $\mathrm{P}_{n}$ can be rewritten as follows:

$$
\mathrm{P}_{n}= \begin{cases}\operatorname{Pr}\left(\chi_{n}>\varphi_{n}\right) ; & \text { if } \kappa_{\mathrm{D} n}=\kappa_{\mathrm{E} n} \\ \operatorname{Pr}\left(\varphi_{n}<\frac{\chi_{n}}{1+\Delta_{1} \chi_{n}}\right) ; & \text { if } \kappa_{\mathrm{D} n}>\kappa_{\mathrm{E} n} \\ \operatorname{Pr}\left(\chi_{n}>\frac{\varphi_{n}}{1+\Delta_{2} \varphi_{n}}\right) ; & \text { if } \kappa_{\mathrm{D} n}<\kappa_{\mathrm{E} n}\end{cases}
$$

where $\Delta_{1}=\kappa_{\mathrm{D} n}-\kappa_{\mathrm{E} n}$ and $\Delta_{2}=\kappa_{\mathrm{E} n}-\kappa_{\mathrm{D} n}$.

As presented in (10), depending on values of $\kappa_{\mathrm{D} n}$ and $\kappa_{\mathrm{E} n}$, we consider three cases as follows:

Case 1: $\kappa_{\mathrm{D} n}=\kappa_{\mathrm{E} n}$

In this case, the authorized node $\mathrm{T}_{n}$ and the eavesdropper $\mathrm{E}$ are assumed to have the same hardware structure (e.g., both are mobile users), hence their hardware impairment levels are same (see [13]). Then, from (10), the PoNSC at the $n$th hop is formulated by

$$
\mathrm{P}_{n}=\int_{0}^{+\infty}\left(1-F_{\chi_{n}}(x)\right) f_{\varphi_{n}}(x) \mathrm{d} x .
$$

Substituting the results given in (4) and (6) into (11); and using [18, eq. (3.351.3)] for the corresponding integrals, we can obtain

$$
\mathrm{P}_{n}=\sum_{t=0}^{m_{\mathrm{D} n}-1} \frac{\left(t+m_{\mathrm{E} n}-1\right) !}{t !\left(m_{\mathrm{E} n}-1\right) !} \frac{\lambda_{n}^{t} \Omega_{n}^{m_{\mathrm{E} n}}}{\left(\lambda_{n}+\Omega_{n}\right)^{t+m_{\mathrm{E} n}}} .
$$

It can be observed from (12) that the PoNSC $\mathrm{P}_{n}$ does not depend on the transmit $\operatorname{SNR} \psi\left(P / N_{0}\right)$ as well as the hardware impairment levels.

Case 2: $\kappa_{\mathrm{D} n}>\kappa_{\mathrm{E} n}$

In this case, the eavesdropper is equipped with a better transceiver, as compared with that of the nodes $T_{n}$, e.g., the node $\mathrm{E}$ is a base station while $\mathrm{T}_{\mathrm{n}}$ is a mobile user. Then, the PoNSC in (10) can be formulated by

$$
\mathrm{P}_{n}=\int_{0}^{+\infty} F_{\varphi_{n}}\left(\frac{x}{1+\Delta_{1} x}\right) f_{\chi_{n}}(x) \mathrm{d} x .
$$

Combining (4), (6) and (13), after some manipulations, it can be obtained that

$$
\begin{aligned}
\mathrm{P}_{n}=1- & \sum_{t=0}^{m_{\mathrm{E} n}-1} \frac{\Omega_{n}^{t}}{t !} \frac{\lambda_{n}^{m_{\mathrm{D} n}}}{\left(m_{\mathrm{D} n}-1\right) !} \\
& \times \int_{0}^{+\infty} \frac{x^{t+m_{\mathrm{D} n}-1}}{\left(1+\Delta_{1} x\right)^{t}} \exp \left(-\frac{\Omega_{n} x}{1+\Delta_{1} x}-\lambda_{n} x\right)
\end{aligned}
$$

Next, by changing variable $y=1+\Delta_{1} x$, we can rewrite (14) under the following form:

$$
\begin{gathered}
\mathrm{P}_{n}=1-\sum_{t=0}^{m_{\mathrm{E} n}-1} \frac{\Omega_{n}^{t}}{t !} \frac{\lambda_{n}^{m_{\mathrm{D} n}}}{\left(m_{\mathrm{D} n}-1\right) !} \frac{1}{\Delta_{1}^{t+m_{\mathrm{D} n}}} \exp \left(\frac{\lambda_{n}-\Omega_{n}}{\Delta_{1}}\right) \\
\times \int_{1}^{+\infty} \frac{(y-1)^{t+m_{\mathrm{D} n}-1}}{y^{t}} \exp \left(\frac{\Omega_{n}}{\Delta_{1} y}\right) \exp \left(-\frac{\lambda_{n}}{\Delta_{1}} y\right) \mathrm{d} y
\end{gathered}
$$


Next, applying binomial expansion for $(y-1)^{t+m_{\mathrm{D} n}-1}$; and substituting this result into (15), we arrive at

$$
\begin{aligned}
\mathrm{P}_{n}=1- & \sum_{t=0}^{m_{\mathrm{E} n}-1} \frac{\Omega_{n}^{t}}{t !} \frac{\lambda_{n}^{m_{\mathrm{D} n}}}{\left(m_{\mathrm{D} n}-1\right) !} \frac{1}{\Delta_{1}^{t+m_{\mathrm{D} n}} \exp \left(\frac{\lambda_{n}-\Omega_{n}}{\Delta_{1}}\right)} \\
& \times \sum_{v=0}^{t+m_{\mathrm{D} n}-1}(-1)^{v} C_{t+m_{\mathrm{D} n}-1}^{v} \\
& \times \int_{1}^{+\infty} y^{m_{\mathrm{D} n}-v-1} \exp \left(\frac{\Omega_{n}}{\Delta_{1} y}\right) \exp \left(-\frac{\lambda_{n}}{\Delta_{1}} y\right) \mathrm{d} y .
\end{aligned}
$$

Remark 1: It can be observed that the PoNSC $\mathrm{P}_{n}$ is expressed by sum of integrals because it is impossible to find a closed-form expression for the integrals given in (16). However, it is too difficult to use integral-form expressions to design and optimize the system, which motivates us to express $\mathrm{P}_{n}$ by a more useful form: infinite series of closed-form expressions (see [21]).

At first, the exponential function $\exp \left(\Omega_{n} / \Delta_{1} / y\right)$ in (16) can be expressed by an infinite series as

$$
\exp \left(\frac{\Omega_{n}}{\Delta_{1} y}\right)=\sum_{w=0}^{+\infty} \frac{1}{w !}\left(\frac{\Omega_{n}}{\Delta_{1} y}\right)^{w} .
$$

Substituting (17) into (16), which yields

$$
\begin{aligned}
\mathrm{P}_{n}=1- & \sum_{t=0}^{m_{\mathrm{E} n}-1} \frac{\Omega_{n}^{t}}{t !} \frac{\lambda_{n}^{m_{\mathrm{D} n}}}{\left(m_{\mathrm{D} n}-1\right) !} \frac{1}{\Delta_{1}^{t+m_{\mathrm{D} n}}} \exp \left(\frac{\lambda_{n}-\Omega_{n}}{\Delta_{1}}\right) \\
& \times \sum_{v=0}^{t+m_{\mathrm{D} n}-1} \sum_{w=0}^{+\infty} \frac{(-1)^{v}}{w !} C_{t+m_{\mathrm{D} n}-1}^{v}\left(\frac{\Omega_{n}}{\Delta_{1}}\right)^{w} \\
& \times \underbrace{\int_{1}^{+\infty} y^{m_{\mathrm{D} n}-v-w-1} \exp \left(-\frac{\lambda_{n}}{\Delta_{1}} y\right) \mathrm{d} y}_{I_{1}}
\end{aligned}
$$

In order to calculate the integral $I_{1}$ in (18), we have to consider three cases as follows:

- $m_{\mathrm{D} n}-w-v-1=0$ or $w=m_{\mathrm{D} n}-v-1$

In this case, it is obvious that

$$
I_{1}=\frac{\Delta_{1}}{\lambda_{n}} \exp \left(-\frac{\lambda_{n}}{\Delta_{1}}\right)
$$

$$
\text { - } m_{\mathrm{D} n}-w-v-1>0 \text { or } w<m_{\mathrm{D} n}-v-1
$$

At first, using [18, 2.321.2], we obtain a following result:

$$
\int_{1}^{+\infty} x^{r} \exp (-a x) \mathrm{d} x=\exp (-a) \sum_{k=0}^{r} k ! C_{r}^{k} / a^{k+1}
$$

where $r$ is a positive integer, $a$ is positive constant and $C_{r}^{k}=r ! / k ! /(r-k) !$. From (20), the integral $I_{1}$ in this case can be calculated by

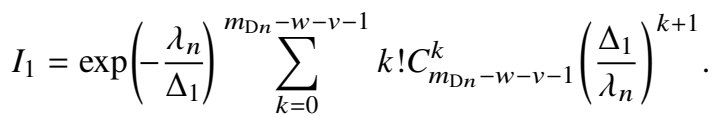

- $m_{\mathrm{D} n}-w-v-1<0$ or $w>m_{\mathrm{D} n}-v-1$

In this case, by employing [18, 3.351.4], we have

$$
\begin{aligned}
\int_{1}^{+\infty} \frac{\exp (-a x)}{x^{r}} \mathrm{~d} x & =\exp (-a) \sum_{k=0}^{r-2} \frac{(-1)^{k} a^{k}}{\prod_{l=0}^{k}(r-1-l)} \\
& +\frac{(-1)^{r-1} a^{r-1}}{(r-1) !} E_{1}(a)
\end{aligned}
$$

where $r$ is a positive integer, $a$ is positive constant and $E_{1}($. is exponential integral function [18]. Therefore, we can calculate $I_{1}$ by

$$
\begin{aligned}
I_{1} & =\int_{1}^{+\infty} \frac{1}{y^{v+w+1-m_{\mathrm{D} n}}} \exp \left(-\frac{\lambda_{n}}{\Delta_{1}} y\right) \mathrm{d} y \\
& =\exp \left(-\frac{\lambda_{n}}{\Delta_{1}}\right)^{w+v-m_{\mathrm{D} n}-1} \sum_{k=0} \frac{(-1)^{k}\left(\lambda_{n} / \Delta_{1}\right)^{k}}{\prod_{l=0}^{k}\left(w+v-m_{\mathrm{D} n}-l\right)} \\
& +\frac{(-1)^{w+v-m_{\mathrm{D} n}}\left(\lambda_{n} / \Delta_{1}\right)^{w+v-m_{\mathrm{D} n}}}{\left(w+v-m_{\mathrm{D} n}\right) !} E_{1}\left(\frac{\lambda_{n}}{\Delta_{1}}\right)
\end{aligned}
$$

From (18), (19), (21) and (23), an exact expression of the PoNSC $\mathrm{P}_{n}$ can be given as in (24).

However, equation (24) is still complex which does not provide any insights into system performance, which motivates us to find simpler expressions for $\mathrm{P}_{n}$.

At first, when the transmit SNR $\psi$ is high, we can approximate $\mathrm{P}_{n}$ in (10) by

$$
\begin{aligned}
\mathrm{P}_{n} & \stackrel{\psi \rightarrow+\infty}{\approx} \operatorname{Pr}\left(\varphi_{n}<\frac{1}{\Delta_{1}}\right)=F_{\varphi_{n}}\left(\frac{1}{\Delta_{1}}\right) \\
& \stackrel{\psi \rightarrow+\infty}{\approx} 1-\exp \left(-\frac{\Omega_{n}}{\Delta_{1}}\right) \sum_{t=0}^{m_{\mathrm{E} n}-1} \frac{1}{t !}\left(\frac{\Omega_{n}}{\Delta_{1}}\right)^{t}
\end{aligned}
$$

Moreover, (25) can be approximated by a simpler expression as follows:

$$
\mathrm{P}_{n} \stackrel{\psi \rightarrow+\infty}{\approx} F_{\varphi_{n}}\left(\frac{1}{\Delta_{1}}\right) \stackrel{\psi \rightarrow+\infty}{\approx} \frac{1}{m_{\mathrm{E} n} !}\left(\frac{\Omega_{n}}{\Delta_{1}}\right)^{m_{\mathrm{E} n}}
$$

Substituting the results obtained by (25) and (26) into (9), the PoNSC can be approximated by the following closedform formulas:

$$
\mathrm{P}_{\mathrm{NSC}} \stackrel{\psi \rightarrow+\infty}{\approx} \prod_{n=1}^{N}\left[1-\exp \left(-\frac{\Omega_{n}}{\Delta_{1}}\right)^{m_{\mathrm{E} n}-1} \sum_{t=0}^{1} \frac{1}{t !}\left(\frac{\Omega_{n}}{\Delta_{1}}\right)^{t}\right],
$$

$$
\mathrm{P}_{\mathrm{NSC}} \stackrel{\psi \rightarrow+\infty}{\approx} \prod_{n=1}^{N} \frac{1}{m_{\mathrm{E} n} !}\left(\frac{\Omega_{n}}{\Delta_{1}}\right)^{m_{\mathrm{E} n}}
$$




$$
\begin{aligned}
& \mathrm{P}_{n}=1-\sum_{t=0}^{m_{\mathrm{E} n}-1} \frac{\Omega_{n}^{t}}{t !} \frac{\lambda_{n}^{m_{\mathrm{D} n}}}{\left(m_{\mathrm{D} n}-1\right) !} \frac{1}{\Delta_{1}^{t+m_{\mathrm{D} n}}} \exp \left(\frac{\lambda_{n}-\Omega_{n}}{\Delta_{1}}\right)
\end{aligned}
$$

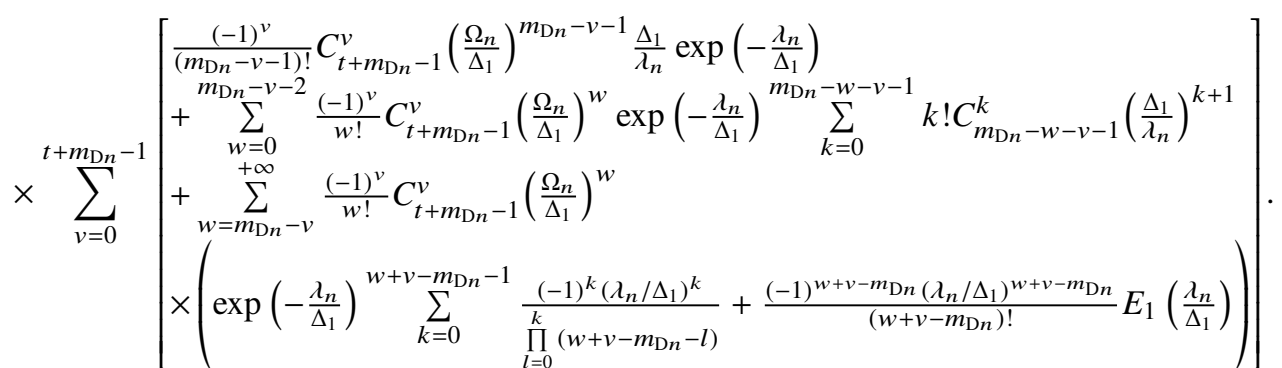

Remark 2: From (25), it is obvious that the $\operatorname{PoSNC~}_{n}$ at high transmit SNR $\psi$ depends on $\psi$ and $m_{\mathrm{E} n}$. In particular, $\mathrm{P}_{n}$ decreases when the value of $\psi$ increases, which means that as $\kappa_{\mathrm{D} n}>\kappa_{\mathrm{E} n}$, the secrecy performance is worse with increasing of the transmit power. Moreover, the performance also degrades with higher value of the Nakagami- $m$ parameter of the eavesdropping link $\left(m_{\mathrm{E} n}\right)$. Indeed, as shown in (26) and (28), the $\mathrm{P}_{n}$ and $\mathrm{P}_{\mathrm{NSC}}$ decrease with the slope of $m_{\mathrm{E} n}$ and $\sum_{n=1}^{N} m_{\mathrm{E} n}$, respectively.

Case 3: $\kappa_{\mathrm{D} n}<\kappa_{\mathrm{E} n}$

In this case, the hardware transceiver of the nodes $T_{n}$ is better that of the eavesdropper, e.g., $\mathrm{T}_{\mathrm{n}}$ can be a base station while $\mathrm{E}$ is only a mobile user. Similarly, we can rewrite $\mathrm{P}_{n}$ in (10) under the following form:

$$
\begin{aligned}
\mathrm{P}_{n} & =\int_{0}^{+\infty}\left(1-F_{\chi_{n}}\left(\frac{x}{1+\Delta_{2} x}\right)\right) f_{\varphi_{n}}(x) \mathrm{d} x \\
& =\sum_{t=0}^{m_{\mathrm{D} n}-1} \frac{\lambda_{n}^{t}}{t !} \frac{\Omega_{n}^{m_{\mathrm{E} n}}}{\left(m_{\mathrm{E} n}-1\right) !} \frac{1}{\Delta_{2}^{t+m_{\mathrm{E} n}}} \exp \left(\frac{\Omega_{n}-\lambda_{n}}{\Delta_{2}}\right) \\
& \times \sum_{v=0}^{t+m_{\mathrm{E} n}-1} \sum_{w=0}^{+\infty} \frac{(-1)^{v}}{w !} C_{t+m_{\mathrm{E} n}-1}^{v}\left(\frac{\lambda_{n}}{\Delta_{2}}\right)^{w} \\
& \times \underbrace{\int_{1}^{+\infty} y^{m_{\mathrm{E} n}-v-w-1} \exp \left(-\frac{\Omega_{i}}{\Delta_{2}} y\right) \mathrm{d} y}_{I_{2}} .
\end{aligned}
$$

Similarly, when $w=m_{\mathrm{E} n}-v-1, w<m_{\mathrm{E} n}-v-1$ and $w>m_{\mathrm{E} n}-v-1$, the integral $I_{2}$ in (29) can be calculated respectively by

$$
\begin{aligned}
I_{2} & =\frac{\Delta_{2}}{\Omega_{n}} \exp \left(-\frac{\Omega_{n}}{\Delta_{2}}\right) \\
I_{2} & =\exp \left(-\frac{\Omega_{n}}{\Delta_{2}}\right)^{m} \sum_{k=0}^{m_{\mathrm{E} n}-w-v-1} k ! C_{m_{\mathrm{E} n}-w-v-1}^{k}\left(\frac{\Delta_{2}}{\Omega_{n}}\right)^{k+1}, \\
I_{2} & =\exp \left(-\frac{\Omega_{n}}{\Delta_{2}}\right)^{w+v-m_{\mathrm{E} n-1}} \sum_{k=0} \frac{(-1)^{k}\left(\Omega_{n} / \Delta_{2}\right)^{k}}{\prod_{l=0}^{k}\left(w+v-m_{\mathrm{E} n}-l\right)} \\
& +\frac{(-1)^{w+v-m_{\mathrm{E} n}}\left(\Omega_{n} / \Delta_{2}\right)^{w+v-m_{\mathrm{E} n}}}{\left(w+v-m_{\mathrm{E} n}\right) !} E_{1}\left(\frac{\Omega_{n}}{\Delta_{2}}\right)
\end{aligned}
$$

From the results obtained above, an exact expression of $\mathrm{P}_{n}$ can be given by (31).

Also, considering the value of $\mathrm{P}_{n}$ at high $\psi$ regions, we obtain the following result:

$$
\begin{aligned}
\mathrm{P}_{n} & \stackrel{\psi \rightarrow+\infty}{\approx} \operatorname{Pr}\left(\gamma_{n}>\frac{1}{\Delta_{2}}\right) \\
& \underset{\sim \rightarrow+\infty}{\approx} \sum_{t=0}^{m_{\mathrm{D} n}-1} \frac{1}{t !}\left(\frac{\lambda_{n}}{\Delta_{2}}\right)^{t} \exp \left(-\frac{\lambda_{n}}{\Delta_{2}}\right) .
\end{aligned}
$$

From (9) and (32), the approximate closed-form expression of the PoNSC at high transmit SNR can be expressed as follows:

$$
\mathrm{P}_{\mathrm{NSC}} \stackrel{\psi \rightarrow+\infty}{\approx} \prod_{n=1}^{N}\left[\sum_{t=0}^{m_{\mathrm{D} n}-1} \frac{1}{t !}\left(\frac{\lambda_{n}}{\Delta_{2}}\right)^{t} \exp \left(-\frac{\lambda_{n}}{\Delta_{2}}\right)\right]
$$

Remark 3: We can observe from (32) and (33) that as $\kappa_{\mathrm{D} n}<\kappa_{\mathrm{E} n}$, the value of PoNSC at the $n$th hop increases when the transmit power increases. Furthermore, the PoNSC performance is also better with high value of the Nakagami- $m$ parameter of the data link.

\section{Simulation Results}

In this section, we perform Monte-Carlo simulations to verify the theoretical derivations provided in Section 3. For each Monte-Carlo simulation, we performed $10^{6}$ trials in which the channel coefficients between two nodes $\mathrm{X}$ and $\mathrm{Y}$ are randomly generated, where $\mathrm{X}, \mathrm{Y} \in\left\{\mathrm{T}_{0}, \mathrm{~T}_{1}, \ldots, \mathrm{T}_{\mathrm{N}}, \mathrm{E}\right\}$. Then, the simulated PNSC is computed by the number of trials that the end-to-end secrecy capacity is higher than zero divided by the number of trials $\left(10^{6}\right)$. In the simulation environment, the source is placed at the position $(0,0)$, the destination is located at $(1,0)$, the position of the relay node $\mathrm{T}_{\mathrm{n}}$ is $(n / N, 0)$, where $n=1,2, \ldots, N-1$, and the position of the eavesdropper is $\left(x_{\mathrm{E}}, y_{\mathrm{E}}\right)$, where $0 \leq x_{\mathrm{E}}, y_{\mathrm{E}} \leq 1$. In all of the simulations, the path-loss exponent $\beta$ is fixed by 3 . In order to present the impact of the hardware imperfection on the secrecy performance clearly, we can assume that the 


$$
\begin{aligned}
& \mathrm{P}_{n}=\sum_{t=0}^{m_{\mathrm{D} n}-1} \frac{\lambda_{n}^{t}}{t !} \frac{\Omega_{n}^{m_{\mathrm{E} n}}}{\left(m_{\mathrm{E} n}-1\right) !} \frac{1}{\Delta_{2}^{t+m_{\mathrm{E} n}}} \exp \left(\frac{\Omega_{n}-\lambda_{n}}{\Delta_{2}}\right)
\end{aligned}
$$

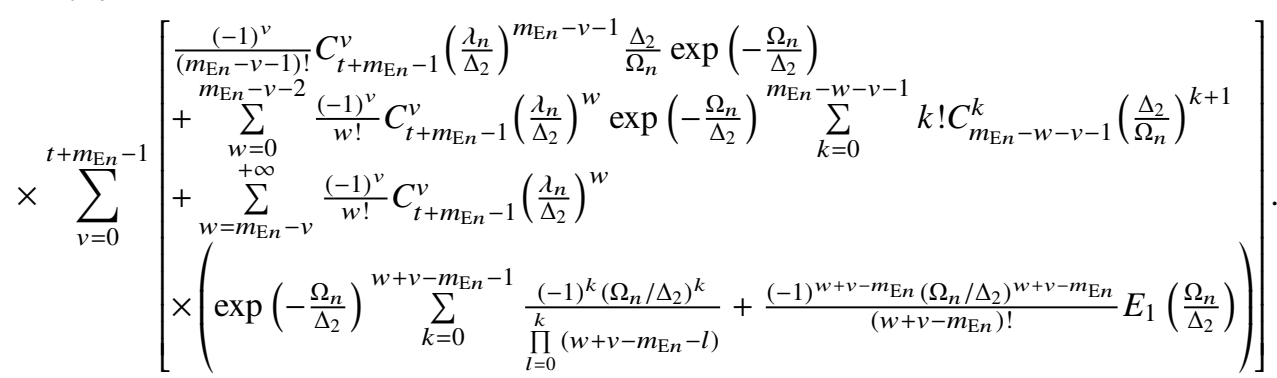

hardware impairment levels on the data links (the eavesdropping links $)$ are same, i.e., $\kappa_{\mathrm{D} n}=\kappa_{\mathrm{D}}\left(\kappa_{\mathrm{E} n}=\kappa_{\mathrm{E}}\right)$ for all $n$. Also, it is also assumed that $m_{\mathrm{D} n}=m_{\mathrm{D}}$ and $m_{\mathrm{E} n}=m_{\mathrm{E}}$ for all $n$. Moreover, for the theoretical results, we truncate infinite series at first 100 terms.

In Fig. 2, we present the PoNSC as a function of the transmit SNR $\psi\left(P / N_{0}\right)$ in $\mathrm{dB}$. In this simulation, the number of hops $(N)$ is fixed by 4 , the Nakagami- $m$ parameters $\mathrm{m}_{\mathrm{D}}$ is set by 2 , the hardware impairment levels $\kappa_{\mathrm{D}}$ and $\kappa_{\mathrm{E}}$ equals to 0.2 and 0.1 , respectively, the eavesdropper is located at the position $(0.5,0.5)$. We can see that the secrecy performance significantly degrades with the increasing of the transmit SNR. Moreover, the PoNSC of the proposed protocol is also worse when the channels of the eavesdropping links are better ( $m_{\mathrm{E}}$ is higher). Finally, it is worth noting that the simulation results (denoted by Sim) match very well with the theoretical ones (denoted by Exact), which validates our derivations.

In Fig. 3, we verify the approximate closed-form expressions given in (27) and (28) by presenting the theoretical results of PoNSC at high transmit SNR. The parameters used for this simulation are $N=5, m_{\mathrm{D}}=2, \kappa_{\mathrm{D}}=0.2, \kappa_{\mathrm{E}}=0$, $x_{\mathrm{E}}=0.3$ and $y_{\mathrm{E}}=0.4$. It can be seen that the asymptotic values (denoted by Appro) in (27) rapidly converges to the exact ones at low and medium $\psi$ region, while that of (28) reaches to the exact ones at medium and high $\psi$ regime. Moreover, we can also observe from this figure that the slope of the curves equals to the number of $N m_{\mathrm{E}}$, which verifies the result obtained in (28).

Figure 4 presents the PoNSC as a function of the transmit SNR $\psi$ in $\mathrm{dB}$ when the hardware transceiver of the authorized nodes is better than that of the eavesdropper, i.e., $\kappa_{\mathrm{D}}<\kappa_{\mathrm{E}}$. In this figure, we set the value of the parameters by $N=3, m_{\mathrm{E}}=3, \kappa_{\mathrm{D}}=0.05, \kappa_{\mathrm{E}}=0.1$ and $x_{\mathrm{E}}=y_{\mathrm{E}}=0.3$. As illustrated in Fig. 4, the PoNSC increases when the transmit SNR $\psi$ increases. In addition, the performance is also better when the Nakagami- $m$ parameter of the data link is higher. Again, the simulation and theoretical results are in good agreement, while the asymptotic PoNSC converges to the exact PoNSC at medium and high $\psi$ regimes.

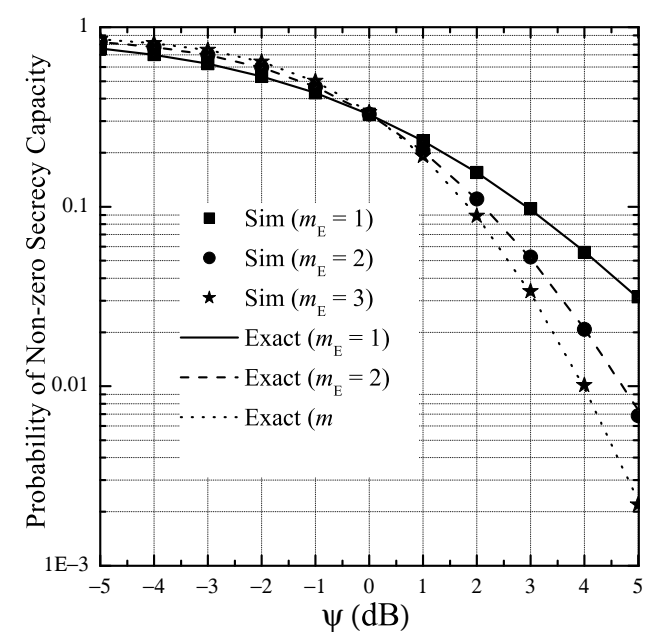

Fig. 2. Probability of non-zero secrecy capacity as a function of the transmit SNR $\psi$ in $\mathrm{dB}$ with different values of $m_{\mathrm{E}}$, when $N=4, m_{\mathrm{D}}=2, \kappa_{\mathrm{D}}=0.2, \kappa_{\mathrm{E}}=0.1$ and $x_{\mathrm{E}}=y_{\mathrm{E}}=0.5$.

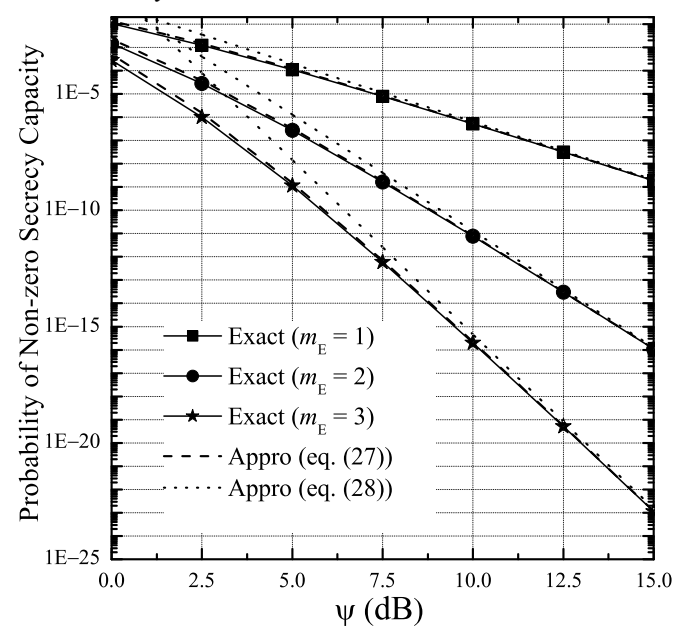

Fig. 3. Probability of non-zero secrecy capacity as a function of the transmit SNR $\psi$ in $\mathrm{dB}$ with different values of $m_{\mathrm{E}}$, when $N=5, m_{\mathrm{D}}=2, \kappa_{\mathrm{D}}=0.2, \kappa_{\mathrm{E}}=0, x_{\mathrm{E}}=0.3$ and $y_{\mathrm{E}}=0.4$.

In Fig. 5, we investigate the impact of the number of hops on the secrecy performance of the proposed protocol when $\psi=0 \mathrm{~dB}, m_{\mathrm{D}}=1, m_{\mathrm{E}}=2, \kappa_{\mathrm{D}}=0.05, \kappa_{\mathrm{E}}=0.1$ and $x_{\mathrm{E}}=y_{\mathrm{E}}=0.5$. An interesting result presented in this figure 


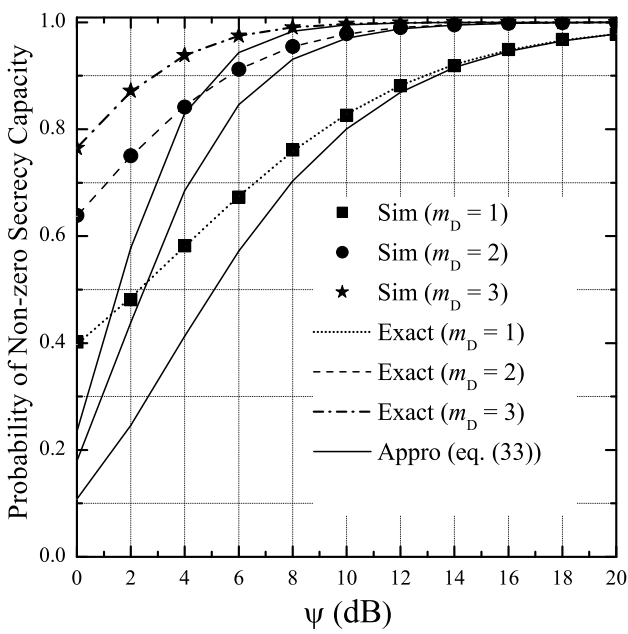

Fig. 4. Probability of non-zero secrecy capacity as a function of the transmit SNR $\psi$ in $\mathrm{dB}$ with different values of $m_{\mathrm{D}}$, when $N=3, m_{\mathrm{E}}=3, \kappa_{\mathrm{D}}=0.05, \kappa_{\mathrm{E}}=0.1$ and $x_{\mathrm{E}}=y_{\mathrm{E}}=0.3$.

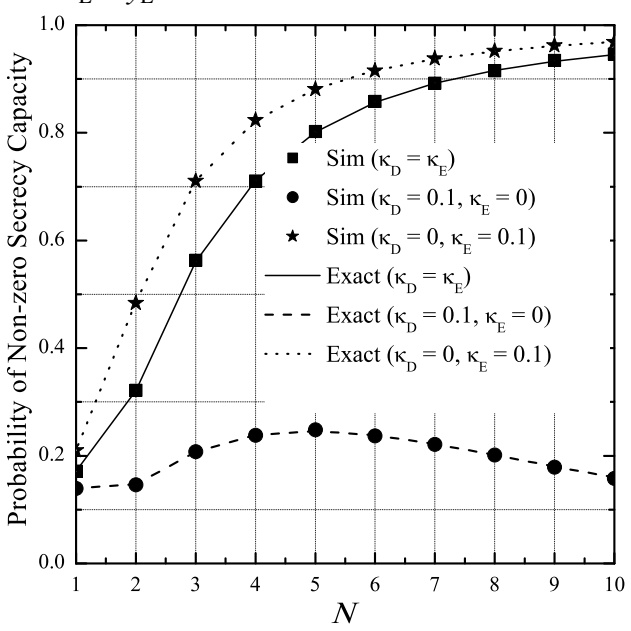

Fig. 5. Probability of non-zero secrecy capacity as a function of the number of hops $N$ with different values of $\kappa_{\mathrm{D}}$ and $\kappa_{\mathrm{E}}$, when $\psi=0 \mathrm{~dB} m_{\mathrm{D}}=1, m_{\mathrm{E}}=2, \kappa_{\mathrm{D}}=0.05, \kappa_{\mathrm{E}}=0.1$ and $x_{\mathrm{E}}=y_{\mathrm{E}}=0.5$.

is that when the hardware impairment level at the authorized nodes is lower or equal to that of the eavesdropper, the PoNSC increases when the number of hops increases. It is also seen that in case where $\kappa_{\mathrm{D}}>\kappa_{\mathrm{E}}$, the secrecy performance changes with the increasing of the number of hops. Moreover, it is worth noting that there exists an optimal value of $N$ at which the PoNSC is highest, i.e., $N=5$.

Figure 6 presents the impact of the hardware impairment level $\kappa_{\mathrm{E}}$ on the PoNSC with different positions of the eavesdropper, i.e., $(0,0.3),(0.5,0.3)$ and $(1,0.3)$. The remaining parameters are set by $\psi=0 \mathrm{~dB}, N=4, m_{\mathrm{D}}=1$, $m_{\mathrm{E}}=2$ and $\kappa_{\mathrm{D}}=0.55$. As expected, the secrecy performance of the proposed protocol is better when the hardware impairment level $\kappa_{\mathrm{E}}$ increases. Moreover, the eavesdropper's position affects on the system performance. For example, in this figure, the PoNSC is highest when the eavesdropper is near the destination $\left.\left(x_{\mathrm{E}}, y_{\mathrm{E}}\right)=(1,0.3)\right)$, and the PoNSC is lowest when the eavesdropper is located at $(0.5,0.3)$.

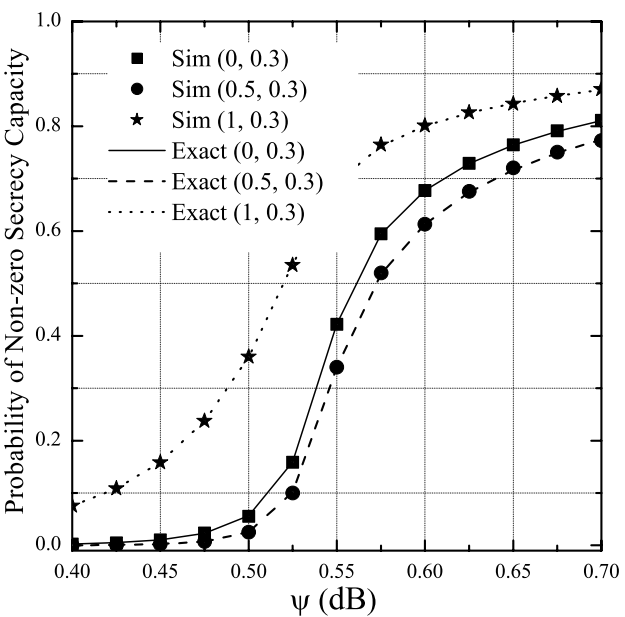

Fig. 6. Probability of non-zero secrecy capacity as a function of $\kappa_{\mathrm{E}}$ with various positions of the eavesdropper when $\psi=0 \mathrm{~dB}, N=4, m_{\mathrm{D}}=1, m_{\mathrm{E}}=2, \kappa_{\mathrm{D}}=0.55$.

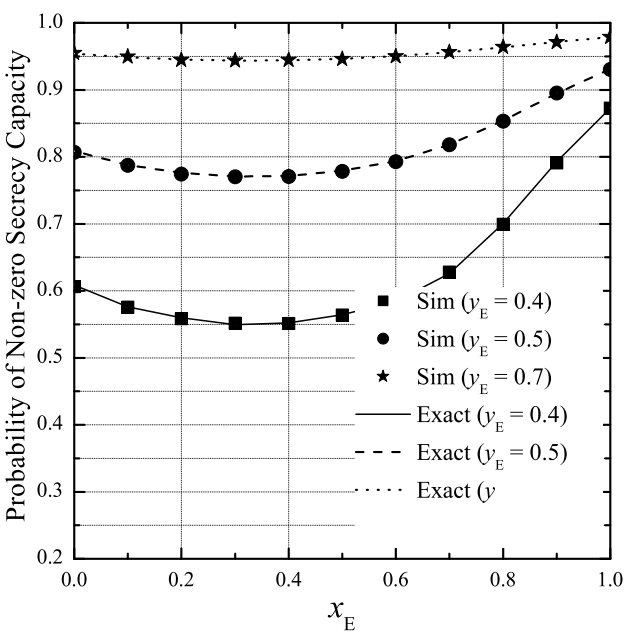

Fig. 7. Probability of non-zero secrecy capacity as a function of $x_{\mathrm{E}}$ with various values of $y_{\mathrm{E}}$ when $N=3, m_{\mathrm{D}}=m_{\mathrm{E}}=2$ and $\kappa_{\mathrm{D}}=\kappa_{\mathrm{E}}$.

In Fig. 7, we investigate the impact of the eavesdropper's position on the secrecy performance when $\kappa_{\mathrm{D}}=\kappa_{\mathrm{E}}, N=3$, $m_{\mathrm{D}}=m_{\mathrm{E}}=2$. In particular, we fix the value of $y_{\mathrm{E}}$, while changing that of $x_{\mathrm{E}}$ from 0 to 1 . As we can see, the secrecy performance significantly improves when the eavesdropper is far the authorized nodes, i.e., $y_{\mathrm{E}}$ increases. Similar to Fig. 6, it is also seen that the PoNSC is highest when the eavesdropper is near the destination. Moreover, for each value of $y_{\mathrm{E}}$, there exists a value of $x_{\mathrm{E}}$ at which the PoNSC is lowest.

\section{Conclusion}

In this paper, the impact of hardware impairments on the probability of non-zero secrecy capacity (PoNSC) of the multi-hop RF relaying protocol was investigated. The obtained results in this paper can be listed as follows:

- We derive exact expressions of the PoNSC over Nakagami- $m$ fading channels. These formulas are expressed by infinite series of exponential functions and 
exponential integral functions. Moreover, Monte Carlo simulations are also performed to validate the correction of our derivations. Results presented that simulation and theoretical results are in good agreement when we truncate infinite series at first 100 terms.

- The hardware impairment level significantly affects on the secrecy performance. In particular, the PoNSC rapidly increases with low impairment level at the authorized nodes and high impairment level at the eavesdropper. In addition, when the transceiver hardware of the eavesdropper is better that of the authorized nodes, the system performance seriously degrades, where the performance-degradation slope equals to the Nakagami- $m$ parameter of the eavesdropping link.

- When the hardware impairment level at the authorized nodes are better than that of the eavesdropper, the PoNSC increases with the increasing of the transmit SNR and the number of hops. Furthermore, with the same impairment levels at all of the nodes, the secrecy performance does not depend on the transmit SNR as well as the hardware impairments.

- The position of the eavesdropper also affects on the value of the PoNSC, i.e., the system performance is better when the eavesdropper is near the destination.

The results obtained in this paper are useful for designing and optimizing the multi-hop secured communication networks. In future works, we will study secured relaying protocols with relay selection methods in presence of hardware impairments.

\section{Acknowledgments}

This research is funded by Vietnam National Foundation for Science and Technology Development (NAFOSTED) under grant number 102.01-2014.33.

\section{References}

[1] WYNER, A. D. The wire-tap channel. The Bell System Technical Journal, 1975, vol. 54, no. 8, p. 1355-1387. DOI: $10.1002 /$ j.1538-7305.1975.tb02040.x

[2] GOPALA, P. K., LAI, L., GAMAL, H. E. On the secrecy capacity of fading channels. IEEE Transactions on Information Theory, 2008, vol. 54, no. 10, p. 4687-4698. DOI: 10.1109/TIT.2008.928990

[3] WANG, L., YANG, N., ELKASHLAN, M., et al. Physical layer security of Maximal ratio combining in two-wave with diffuse power fading channels. Transactions on Information Forensics and Security, 2014, vol. 9, no. 2 , p. 247 - 258. DOI: 10.1109/TIFS.2013.2296991

[4] KAKITANI, M., BRANTEB, G., SOUZAS, R. Energy efficiency analysis of a two dimensional cooperative wireless sensor network with relay selection. Radioengineering, 2013, vol. 22, no. 2, p. 549-557. ISSN: 1805-9600
[5] WANG, G., GUO, D., LIU, A., et al. Multiuser cooperation with hybrid network coding in wireless networks. Radioengineering, 2014, vol. 23, no. 1, p. 435-444. ISSN: 1805-9600

[6] LIU, Y., WANG, L., DUY, T. T., et al. Relay selection for security enhancement in cognitive relay networks. IEEE Wire less Communications Letters, 2015, vol. 4, no. 1, p. 46-49. DOI: 10.1109/LWC.2014.2365808

[7] DUY, T. T., DUONG, T. Q., THANH, T. L., et al. Secrecy performance analysis with relay selection methods under impact of cochannel interference. IET Communications, 2015, vol. 9, no. 11, p. 1427-1435. DOI: $10.1049 /$ iet-com.2014.1128

[8] FAN, L., ZHANG, S., DUONG, T. Q., et al. Secure switchand-stay combining (SSSC) for cognitive relay networks. IEEE Transactions on Communications, 2016, vol. 64, no. 1, p. 70-82. DOI: 10.1109/TCOMM.2015.2497308

[9] DING, Z., MA, Z., FAN, P. Asymptotic studies for the impact of antenna selection on secure two-way relaying communications with artificial noise. IEEE Transactions on Wireless Communications, 2014, vol. 13, no. 4, p. 2189-2203. DOI: 10.1109/TWC.2014.022714131252

[10] DUY, T. T., KONG, H. Y. Secrecy performance analysis of multihop transmission protocols in cluster networks. Wireless Personal Communications, 2015, vol. 82, no. 4, p. 2505-2518. DOI: $10.1007 / \mathrm{s} 11277-015-2361-y$

[11] SANG, N. Q., KONG, H. Y. Exact outage analysis of the effect of cochannel interference on secured multi-hop relaying networks. International Journal of Electronics, 2016, vol. 103, no. 11, p. 1822-1838. DOI: $10.1080 / 00207217.2016 .1138534$

[12] BJORNSON, E., MATTHAiOU, M., DEBBAH, M. A new look at dual-hop relaying: Performance limits with hardware impairments. IEEE Transactions on Communications, 2013, vol. 61, no. 11, p. 4512-4525. DOI: 10.1109/TCOMM.2013.100913.130282

[13] DUY, T. T., DUONG, T. Q., DA COSTA, D. B., et al. Proactive relay selection with joint impact of hardware impairment and co-channel interference. IIEEE Transactions on Communications, 2015, vol. 63, no. 5, p. 1594-1606. DOI: 10.1109/TCOMM.2015.2396517

[14] BOULOGEORGOS, A. A., KARAS, D. S., KARAGIANNIDIS, G. K. How much does I/Q Imbalance affect secrecy capacity? IEEE Communications Letters, 2016, vol. 20, no. 7, p. 1305-1308. DOI: 10.1109/LCOMM.2016.2558561

[15] MO, J., TAO, M., LIU, Y. Relay placement for physical layer security: A secure connection perspective. IEEE Communications Letters, 2012, vol. 16, no. 6, p. 878-881. DOI: 10.1109/LCOMM.2012.042312.120582

[16] DENG, Y., WANG, L., ELKASLAN, M., et al. Generalized selection combining for cognitive relay networks over Nakagami- $m$ fading. IEEE Transactions on Signal Processing, 2015, vol. 63, no. 8, p. 1993-2006. DOI: 10.1109/TSP.2015.2405497

[17] LEE, S., LEE, H., CHOI, H. H., et al. Outage probability of decodeand-forward relaying systems with efficient partial relay selection in Nakagami fading channels. ETRI Journal, 2014, vol. 36, no. 1, p. 22-30. DOI: 10.4218 /etrij.13.0113.0348

[18] GRADSHTEYN, I., RYZHIKR, I. Table of Integrals, Series, and Products. 7th ed. New York (USA): Academic Press, Inc., 2007. ISBN: 978-0123736376

[19] YANG, N., ELKASLAN, M., YUAN, J. Outage probability of multiuser relay networks in Nakagami- $m$ fading channels. IEEE Transactions on Vehicular Technology, 2010, vol. 59, no. 5, p. 2120-2132. DOI: $10.1109 /$ TVT.2010.2042828 
[20] LANEMAN, J. N., TSE, D. N. C., WORNELL, G. W. Cooperative diversity in wireless networks: Efficient protocols and outage behavior. IEEE Transactions on Information Theory, 2004, vol. 50, no. 12, p. 3062 - 3080. DOI: 10.1109/TIT.2004.838089

[21] ALVI, S. H., WYNE, S. Error analysis of fixed-gain AF relaying with MRC over Nakagami- $m$ fading channels. Radioengineering, 2016, vol. 25 , no. 1, p. 106-113. DOI: 10.13164/re.2016.0106

\section{About the Authors...}

Tran Tin PHU was born in Khanh Hoa, Vietnam, in 1979. He received the Bachelor degree (2002) and Master degree (2008) from Ho Chi Minh City University of Science. In 2007, he was lecturer at the Faculty of Electronics Technology (FET), Industrial University of Ho ChiMinh City. Since 2015, he has been participating in Ph.D program, that had been linked between Technical University of Ostrava, Czech Republic and Ton Duc Thang University, Ho Chi Minh City. His major research interests are wireless communication in $5 \mathrm{G}$, energy harvesting, performance of cognitive radio and physical layer security.

The Hung DANG was born in Ha Tinh, Vietnam, in 1983. He received the B.E. degree in Telecommunication Technological Command from Telecommunications University (TCU), Nha Trang, Khanh Hoa, in 2006 and M.Eng. degree in Telecommunications Engineering from Posts and Telecommunications Institute of Technology (PTIT), Ho Chi Minh city, Vietnam, in 2014, respectively. He is currently a Lecturer in the Faculty of Telecommunications Professional of Telecommunications University. His major research interests are Cooperative Communications, Cognitive Radio, and Physical Layer Security.
Trung Duy TRAN (corresponding author) was born in Nha Trang city, Vietnam, in 1984. He received the B.E. degree in Electronics and Telecommunications Engineering from the French-Vietnamese training program for excellent engineers (PFIEV), Ho Chi Minh City University of Technology, Vietnam in 2007. In 2013, he received the Ph.D degree in electrical engineering from University of Ulsan, South Korea. In 2013, he joined the Department of Telecommunications, Posts and Telecommunications Institute of Technology (PTIT), as a lecturer. His major research interests are cooperative communications, cognitive radio, and physical layer security.

Miroslav VOZNAK obtained his Ph.D. in Telecommunications engineering in 2002 from the Faculty in Electrical Engineering and Computer Science, VSB - Technical University of Ostrava and was appointed as an Associate Professor after his habilitation in the same faculty in 2009. Since 2013, he has been leading a Department of Telecommunications in the VSB - Technical University of Ostrava in position of the department chair. He is an IEEE Senior member, actively enganged as a member in numerous conference programme committees and serving as a member of editorial boards in several journals such as Journal of Communications (US), Advances in Electrical and Electronic Engineering (CZ), Communications (TR), etc. He participated in more than fifteen national and three european projects and since 2011 he has been included as a senior researcher into a Czech National Centre of Excellence (National supercomputing centre). His research interests are focused generally on information and communications technology, particularly on Voice over IP, Quality of Experience, Network security, Wireless networks and last several years on Big Data analytics in mobile cellular networks as well. 